\title{
Application of Extended Homotopy Analysis Method to the Two-Degree-of-Freedom Coupled van der Pol-Duffing Oscillator
}

\author{
Y. H. Qian, S. M. Chen, and L. Shen \\ College of Mathematics, Physics and Information Engineering, Zhejiang Normal University, Jinhua, Zhejiang 321004, China \\ Correspondence should be addressed to Y. H. Qian; qyh2004@zjnu.cn
}

Received 19 January 2014; Accepted 21 February 2014; Published 30 March 2014

Academic Editor: Jinlu Li

Copyright ( 2014 Y. H. Qian et al. This is an open access article distributed under the Creative Commons Attribution License, which permits unrestricted use, distribution, and reproduction in any medium, provided the original work is properly cited.

\begin{abstract}
The extended homotopy analysis method (EHAM) is presented to establish the analytical approximate solutions for two-degreeof-freedom (2-DOF) coupled van der Pol-Duffing oscillator. Meanwhile, the comparisons between the results of the EHAM and standard Runge-Kutta numerical method are also presented. The results demonstrate that the analytical approximate solutions of the EHAM agree well with the numerical integration solutions. For EHAM as an analytical approximation method, we are not sure whether it can apply to all of the nonlinear systems; we can only verify its effectiveness through specific cases. As a result of the existence of nonlinear terms, we must study different types of systems, no matter from the complication of calculation and physical significance.
\end{abstract}

\section{Introduction}

Mathematical methods for the natural and engineering sciences problems have drawn considerable attention in recent years. In normal circumstances, most of the nonlinear dynamical models can be governed by a set of differential equations and auxiliary conditions from modeling processes [1]. Numerous analytical methods have been developed to deal with the nonlinear differential equations, such as the modified perturbation methods [2-5], improved harmonic balance methods $[6,7]$, energy balance method $[8,9]$, and the frequency-amplitude formulation [10, 11]. Enlightening from the basic concepts of the homotopy in topology $[12,13]$, Liao developed the homotopy analysis method (HAM) [1416] which does not require small parameters as one of the efficient analytical techniques in solving a variety of nonlinear vibration problems.

Recently, great attention is paid to the discussion of coupled oscillators of nonlinear dynamical systems because most of practical engineering problems can be governed by such coupled systems [17-19]. The extended homotopy analysis method (EHAM) is one method based on the HAM envisioned first by Liao [16]. More recently, Qian et al. [20] extended the HAM to deal with strongly nonlinear coupled van der Pol oscillators. For EHAM as an analytical approximation method, we are not sure whether it can apply to all of the nonlinear systems; we can only verify its effectiveness through specific cases. As a result of the existence of nonlinear terms, we must study different types of systems, no matter from the complication of calculation and physical significance. By solving such example, it is illustrated that the present techniques are not an adhoc approach; it can be generalized to investigate more complicated nonlinear multi-degree-of-freedom (MDOF) dynamical systems.

In the present work, the exact analytical series solutions of the two-degree-of-freedom (2-DOF) coupled van der PolDuffing system are obtained by using the EHAM, and we also establish the comparisons between the results of the EHAM and standard Runge-Kutta numerical method. It is shown that the periodic solutions of the EHAM are in excellent agreement with the numerical integration ones, even if time $t$ progresses to a certain large domain. In what follows, Section 2 presents the EHAM of the MDOF dynamical system. Moreover, the EHAM is presented to establish the analytical approximate solutions for 2-DOF coupled van der Pol-Duffing oscillator in the next section. In Section 4, numerical comparisons are carried out to authenticate the 
correctness and accuracy of the present method. Finally, the paper ends with concluding remarks in Section 5.

\section{The Extended Homotopy Analysis Method}

The MDOF dynamical system is considered by the following equation:

$$
M \ddot{q}+G \dot{q}+K q=F(\dot{q}, q, t),
$$

where $q$ is an $n$-dimensional unknown vector, a dot denotes the derivative with respect to time $t, M, G$, and $K$ are, respectively, $n \times n$ mass, damping, and stiffness matrixes, and $F$ is the vector function of $\dot{q}, q$, and $t$. Let $F(\dot{q}, q, t) \equiv 0$; then (1) is an autonomous dynamical system.

From (1), we define a nonlinear operator as

$$
\begin{aligned}
N[u(r, t)]= & M \frac{\partial^{2} u(r, t)}{\partial t^{2}}+G \frac{\partial u(r, t)}{\partial t} \\
& +K u(r, t)-F\left(\frac{\partial u}{\partial t}, u, t\right),
\end{aligned}
$$

where $u(r, t)$ is an unknown vector value function and $r$ and $t$ are spatial and temporal variables, respectively.

In (2), the unknown vector functions of $u(r, t)$, $\partial u(r, t) / \partial t$, and $\partial^{2} u(r, t) / \partial t^{2}$ are, respectively,

$$
\begin{gathered}
u(r, t)=\left(x_{1}(t), \ldots, x_{n}(t)\right)^{T}, \\
\frac{\partial u(r, t)}{\partial t}=\left(\frac{d x_{1}}{d t}, \ldots, \frac{d x_{n}}{d t}\right)^{T}, \\
\frac{\partial^{2} u(r, t)}{\partial t^{2}}=\left(\frac{d^{2} x_{1}}{d t^{2}}, \ldots, \frac{d^{2} x_{n}}{d t^{2}}\right)^{T} .
\end{gathered}
$$

According to the fundamental concepts and working procedures of the HAM $[1,2]$, the zeroth-order deformation equation can be constructed as follows:

$$
\begin{aligned}
& (1-p)\left\{L\left[\Phi(r, t ; p)-u_{0}(r, t)\right]\right\} \\
& =p \hbar_{1} H_{1}(t) N[\Phi(r, t ; p)]+\hbar_{2} H_{2}(t) \Pi[\Phi(r, t ; p)],
\end{aligned}
$$

where $p \in[0,1]$ is an embedding parameter, $u_{0}(r, t)$ is the solution of initial guess, $L$ is an auxiliary linear operator, and $\hbar_{i}$ and $H_{i}(t)$ are the auxiliary parameters and the functions, respectively.

The operator $\Pi[\Phi(r, t ; p)]$ has the following property:

$$
\Pi[\Phi(r, t ; 0)]=\Pi[\Phi(r, t ; 1)]=0 .
$$

When $p=0$ and $p=1$, the zeroth-order deformation equation $(4)$ is $\Phi(r, t ; 0)=u_{0}(r, t)$ and $\Phi(r, t ; 1)=u(r, t)$, respectively. Hence, as $p$ increases from 0 to 1 , the solution
$\Phi(r, t ; p)$ varies from the initial guess solution $u_{0}(r, t)$ to the exact solution $u(r, t)$. In this paper,

$$
\begin{aligned}
& \Pi=(1-p) \\
& \quad \times\left[\left(\begin{array}{c}
A(p) \cos \tau+B(p) \sin \tau \\
C(p) \cos \tau
\end{array}\right)\right. \\
& \left.\quad+\left(\begin{array}{c}
\left(\Omega^{2}(p)-\omega_{0}^{2}\right)(\cos \tau+\sin \tau) \\
\left(\Omega^{2}(p)-\omega_{0}^{2}\right) \sin \tau
\end{array}\right)\right],
\end{aligned}
$$

where

$$
\begin{aligned}
& A(p)=\sum_{i=1}^{\infty} a_{i} p^{i}, \quad B(p)=\sum_{i=1}^{\infty} b_{i} p^{i}, \\
& C(p)=\sum_{i=1}^{\infty} c_{i} p^{i},
\end{aligned}
$$

with the initial conditions

$$
\begin{array}{ll}
\Phi_{1}(0, p)=a_{0}+A(p), & \left.\frac{\partial \Phi_{1}(\tau, p)}{\partial \tau}\right|_{\tau=0}=b_{0}+B(p), \\
\Phi_{2}(0, p)=c_{0}+C(p), & \left.\frac{\partial \Phi_{2}(\tau, p)}{\partial \tau}\right|_{\tau=0}=d_{0} .
\end{array}
$$

Setting

$$
u_{m}(r, t)=\left.\frac{1}{m !} \frac{\partial^{m} \Phi(r, t ; p)}{\partial p^{m}}\right|_{p=0},
$$

and expanding $\Phi(r, t ; p)$ into the Taylor series expansion with respect to $p$ in accordance with the theorem of vector-valued function, we obtain

$$
\Phi(r, t ; p)=u_{0}(r, t)+\sum_{m=1}^{+\infty} u_{m}(r, t) p^{m}
$$

If the auxiliary linear operator, initial guess solution, auxiliary parameters $\hbar_{i}$, and auxiliary functions $H_{i}(t)$ are properly chosen, the series equation (10) converges at $p=1$, and we arrive at

$$
u(r, t)=u_{0}(r, t)+\sum_{m=1}^{+\infty} u_{m}(r, t) .
$$

For brevity, the vector of $\mathbf{u}_{m}$ is defined as

$$
\mathbf{u}_{m}=\left\{u_{0}(r, t), u_{1}(r, t), \ldots, u_{m}(r, t)\right\} .
$$

Differentiating the zeroth-order deformation equation (4) $m$ times with respect to $p$ then dividing the equation by $m$ ! and setting $p=0$ yield

$$
\begin{aligned}
& L\left[u_{m}(r, t)-\chi_{m} u_{m-1}(r, t)\right] \\
& =\hbar_{1} H_{1}(t) R_{m}\left(\mathbf{u}_{m-1}, r, t\right)+\hbar_{2} H_{2}(t) \Delta_{m}(r, t),
\end{aligned}
$$


where

$$
\begin{gathered}
\chi_{m}= \begin{cases}0, & m \leq 1 \\
1, & m>1,\end{cases} \\
R_{m}\left(\mathbf{u}_{m-1}, r, t\right)=\left.\frac{1}{(m-1) !} \frac{\partial^{m-1} N(\Phi(r, t ; p))}{\partial p^{m-1}}\right|_{p=0}, \\
\Delta_{m}(r, t)=\left.\frac{1}{m !} \frac{\partial^{m} \Pi(\Phi(r, t ; p))}{\partial p^{m}}\right|_{p=0} .
\end{gathered}
$$

The $m$ th-order deformation equation (13) is a linear equation, which can be readily solved by the symbolic software such as Mathematica.

\section{Application of the EHAM}

In this section, we apply the EHAM for analysis of the two coupled van der Pol-Duffing oscillators:

$$
\begin{aligned}
& \ddot{x}_{1}+\varepsilon \eta_{1}\left(x_{1}^{2}-1\right) \dot{x}_{1}+x_{1}+\varepsilon \alpha_{1} x_{1}^{3}+\varepsilon \delta_{1} x_{1} x_{2}^{2}=0, \\
& \ddot{x}_{2}+\varepsilon \eta_{2}\left(x_{2}^{2}-1\right) \dot{x}_{2}+x_{2}+\varepsilon \alpha_{2} x_{2}^{3}+\varepsilon \delta_{2} x_{2} x_{1}^{2}=0,
\end{aligned}
$$

where the superscript denotes the differentiation with respect to time $t, x_{1}(t)$ and $x_{2}(t)$ are the unknown real functions, and $\varepsilon, \alpha_{1}, \alpha_{2}, \eta_{1}, \eta_{2}, \delta_{1}$, and $\delta_{2}$ are parameters.

We introduce a new variable $\tau$ and substitute $\tau=\omega t$, $x_{1}(t)=u_{1}(\tau)$, and $x_{2}(t)=u_{2}(\tau)$ into (15a) and (15b). Therefore, we have

$$
\begin{gathered}
\omega^{2} u_{1}^{\prime \prime}+\omega \varepsilon \eta_{1}\left(u_{1}^{2}-1\right) u_{1}^{\prime}+u_{1}+\varepsilon \alpha_{1} u_{1}^{3}+\varepsilon \delta_{1} u_{1} u_{2}^{2}=0, \\
\omega^{2} u_{2}^{\prime \prime}+\omega \varepsilon \eta_{2}\left(u_{2}^{2}-1\right) u_{2}^{\prime}+u_{2}+\varepsilon \alpha_{2} u_{2}^{3}+\varepsilon \delta_{2} u_{2} u_{1}^{2}=0,
\end{gathered}
$$

subject to the initial conditions

$$
u_{1}(0)=a, \quad u_{1}^{\prime}(0)=b, \quad u_{2}(0)=c, \quad u_{2}^{\prime}(0)=0,
$$

where a prime denotes the derivative with respect to variable $\tau$. Provided that the periodic solutions in (16a) and (16b) can be expressed by a set of base functions

$$
\{\cos (k \tau), \sin (k \tau) \mid k=0,1,2, \ldots\},
$$

one obtains

$$
\begin{aligned}
& u_{1}(\tau)=\sum_{k=0}^{+\infty}\left(a_{1, k} \cos k \tau+b_{1, k} \sin k \tau\right), \\
& u_{2}(\tau)=\sum_{k=0}^{+\infty}\left(a_{2, k} \cos k \tau+b_{2, k} \sin k \tau\right) .
\end{aligned}
$$

For the initial approximation, $u_{1,0}(\tau)$ and $u_{2,0}(\tau)$ are assumed as

$$
u_{1,0}(\tau)=a_{0} \cos \tau+b_{0} \sin \tau, \quad u_{2,0}(\tau)=c_{0} \cos \tau,
$$

and the linear operator is defined as

$$
L\left(\begin{array}{c}
\Phi_{1}(\tau, p) \\
\Phi_{2}(\tau, p)
\end{array}\right)=\omega_{0}^{2}\left(\begin{array}{l}
\frac{\partial^{2} \Phi_{1}(\tau, p)}{\partial \tau^{2}}+\Phi_{1}(\tau, p) \\
\frac{\partial^{2} \Phi_{2}(\tau, p)}{\partial \tau^{2}}+\Phi_{2}(\tau, p)
\end{array}\right) .
$$

We can define a nonlinear operator as the following by EHAM:

$$
N\left(\begin{array}{c}
\Phi_{1}(\tau, p) \\
\Phi_{2}(\tau, p)
\end{array}\right)=\left(\begin{array}{c}
\Omega^{2}(p) \frac{\partial^{2} \Phi_{1}(\tau, p)}{\partial \tau^{2}}+\varepsilon \eta_{1} \Omega(p)\left[\Phi_{1}{ }^{2}(\tau, p)-1\right] \frac{\partial \Phi_{1}(\tau, p)}{\partial \tau} \\
+\Phi_{1}(\tau, p)+\varepsilon \alpha_{1} \Phi_{1}{ }^{3}(\tau, p)+\varepsilon \delta_{1} \Phi_{1}(\tau, p) \Phi_{2}{ }^{2}(\tau, p) \\
\Omega^{2}(p) \frac{\partial^{2} \Phi_{2}(\tau, p)}{\partial \tau^{2}}+\varepsilon \eta_{2} \Omega(p)\left[\Phi_{2}{ }^{2}(\tau, p)-1\right] \frac{\partial \Phi_{2}(\tau, p)}{\partial \tau} \\
+\Phi_{2}(\tau, p)+\varepsilon \alpha_{2} \Phi_{2}{ }^{3}(\tau, p)+\varepsilon \delta_{2} \Phi_{1}{ }^{2}(\tau, p) \Phi_{2}(\tau, p)
\end{array}\right)
$$

and the nonlinear operator $\Pi$ is

$$
\begin{aligned}
\Pi\left(\begin{array}{c}
\Phi_{1}(\tau, p) \\
\Phi_{2}(\tau, p)
\end{array}\right)= & (1-p) \\
& \times\left[\left(\begin{array}{c}
A(p) \cos \tau+B(p) \sin \tau \\
C(p) \cos \tau
\end{array}\right)\right. \\
& \left.+\left(\begin{array}{c}
\left(\Omega^{2}(p)-\omega_{0}^{2}\right)(\cos \tau+\sin \tau) \\
\left(\Omega^{2}(p)-\omega_{0}^{2}\right) \sin \tau
\end{array}\right)\right] .
\end{aligned}
$$

In terms of the principle of solution expression, we select the auxiliary functions as $H_{1}(t)=1$ and $H_{2}(t)=1$; thus the zeroth-order deformation equation is given by

$$
\begin{aligned}
& (1-p) L\left(\begin{array}{l}
\Phi_{1}(\tau, p)-u_{1,0}(\tau) \\
\Phi_{2}(\tau, p)-u_{2,0}(\tau)
\end{array}\right) \\
& \quad=p \hbar_{1} N\left(\begin{array}{l}
\Phi_{1}(\tau, p) \\
\Phi_{2}(\tau, p)
\end{array}\right)+(1-p)
\end{aligned}
$$




$$
\begin{aligned}
& \times \hbar_{2}\left[\left(\begin{array}{c}
A(p) \cos \tau+B(p) \sin \tau \\
C(p) \cos \tau
\end{array}\right)\right. \\
& \left.\quad+\left(\begin{array}{c}
\left(\Omega^{2}(p)-\omega_{0}^{2}\right)(\cos \tau+\sin \tau) \\
\left(\Omega^{2}(p)-\omega_{0}^{2}\right) \sin \tau
\end{array}\right)\right],
\end{aligned}
$$

where

$$
A(p)=\sum_{i=1}^{\infty} a_{i} p^{i}, \quad B(p)=\sum_{i=1}^{\infty} b_{i} p^{i}, \quad C(p)=\sum_{i=1}^{\infty} c_{i} p^{i}
$$

with the initial conditions

$$
\begin{gathered}
\Phi_{1}(0, p)=a_{0}+A(p),\left.\quad \frac{\partial \Phi_{1}(\tau, p)}{\partial \tau}\right|_{\tau=0}=b_{0}+B(p) \\
\Phi_{2}(0, p)=c_{0}+C(p),\left.\quad \frac{\partial \Phi_{2}(\tau, p)}{\partial \tau}\right|_{\tau=0}=0
\end{gathered}
$$

For $p=0$, the solutions of $(24)-((26 a),(26 b))$ are

$$
\Phi_{1}(\tau, 0)=u_{1,0}(\tau), \quad \Phi_{2}(\tau, 0)=u_{2,0}(\tau), \quad \Omega(0)=\omega_{0}
$$

While $p=1$, the zeroth-order deformation equations $(24)-((26 a),(26 b))$ are equivalent to the original equations (16a), (16b), and (17). Thus, we get

$$
\Phi_{1}(\tau, 1)=u_{1}(\tau), \quad \Phi_{2}(\tau, 1)=u_{2}(\tau), \quad \Omega(1)=\omega .
$$

Obviously, as the embedding parameter $p$ varies from 0 to $1, \Phi_{i}(\tau, p)$ changes from the initial guess $u_{i, 0}(\tau)$ to the exact solutions $u_{i}(\tau)(i=1,2)$. In addition, $\Omega(p)$ changes from the initial guess frequency $\omega_{0}$ to the nonlinear physical frequency $\omega$.

With the help of the Taylor series expansion and (13), we obtain

$$
\begin{gathered}
\Phi_{1}(\tau, p)=u_{1,0}(\tau)+\sum_{m=1}^{+\infty} u_{1, m}(\tau) p^{m} \\
\Phi_{2}(\tau, p)=u_{2,0}(\tau)+\sum_{m=1}^{+\infty} u_{2, m}(\tau) p^{m} \\
\Omega(p)=\omega_{0}+\sum_{m=1}^{+\infty} \omega_{m} p^{m}
\end{gathered}
$$

where

$$
\begin{array}{ll}
u_{1, m}(\tau)=\left.\frac{1}{m !} \frac{\partial^{m} \Phi_{1}(\tau, p)}{\partial p^{m}}\right|_{p=0}, & \\
u_{2, m}(\tau)=\left.\frac{1}{m !} \frac{\partial^{m} \Phi_{2}(\tau, p)}{\partial p^{m}}\right|_{p=0}, \quad \omega_{m}=\left.\frac{1}{m !} \frac{\partial^{m} \Omega(p)}{\partial p^{m}}\right|_{p=0} .
\end{array}
$$

If the auxiliary parameters $\hbar_{1}$ and $\hbar_{2}$ are properly chosen, the power series solutions in (29a), (29b), and (29c) are converged at $p=1$. Then from (28), we get

$$
\begin{aligned}
& u_{1}(\tau)=u_{1,0}(\tau)+\sum_{m=1}^{+\infty} u_{1, m}(\tau), \\
& u_{2}(\tau)=u_{2,0}(\tau)+\sum_{m=1}^{+\infty} u_{2, m}(\tau), \\
& \omega=\omega_{0}+\sum_{m=1}^{+\infty} \omega_{m} .
\end{aligned}
$$

For simplicity, the following vectors are defined as

$$
\begin{aligned}
\vec{u}_{1, n}= & \left\{u_{1,0}(\tau), u_{1,1}(\tau), \ldots, u_{1, n}(\tau)\right\} \\
\vec{u}_{2, n}= & \left\{u_{2,0}(\tau), u_{2,1}(\tau), \ldots, u_{2, n}(\tau)\right\} \\
& \vec{\omega}_{n}=\left\{\omega_{0}, \omega_{1}, \ldots, \omega_{n}\right\} .
\end{aligned}
$$

By differentiating the zeroth-order deformation equation (24) $m$ times with respect to $p$, then dividing the equation by $m$ !, and setting $p=0$, the $m t h$-order deformation equation is formulated as follows:

$$
\begin{aligned}
L\left(\begin{array}{l}
u_{1, m}(\tau)-\chi_{m} u_{1, m-1}(\tau) \\
u_{2, m}(\tau)-\chi_{m} u_{2, m-1}(\tau)
\end{array}\right)= & \hbar_{1}\left(\begin{array}{l}
R_{1, m}\left(\vec{u}_{1, m-1}, \vec{\omega}_{m-1}\right) \\
R_{2, m}\left(\vec{u}_{2, m-1}, \vec{\omega}_{m-1}\right)
\end{array}\right) \\
& +\hbar_{2}\left(\begin{array}{c}
S_{1, m}\left(\tau, \vec{\omega}_{m}\right) \\
S_{2, m}\left(\tau, \vec{\omega}_{m}\right)
\end{array}\right),
\end{aligned}
$$

with the initial conditions

$$
\begin{aligned}
& u_{1, m}(0)=a_{m}, u_{1, m}^{\prime}(0)=b_{m}, \\
& u_{2, m}(0)=c_{m}, u_{2, m}^{\prime}(0)=0 \\
&(m \geq 1),
\end{aligned}
$$

in which

$$
\begin{aligned}
& \left(\begin{array}{l}
R_{1, m}\left(\vec{u}_{1, m-1}, \vec{\omega}_{m-1}\right) \\
R_{2, m}\left(\vec{u}_{2, m-1}, \vec{\omega}_{m-1}\right)
\end{array}\right) \\
& \quad=\left.\frac{1}{(m-1) !} \frac{\partial^{m-1}}{\partial p^{m-1}} N\left(\begin{array}{c}
\Phi_{1}(\tau, p) \\
\Phi_{2}(\tau, p)
\end{array}\right)\right|_{p=0},
\end{aligned}
$$




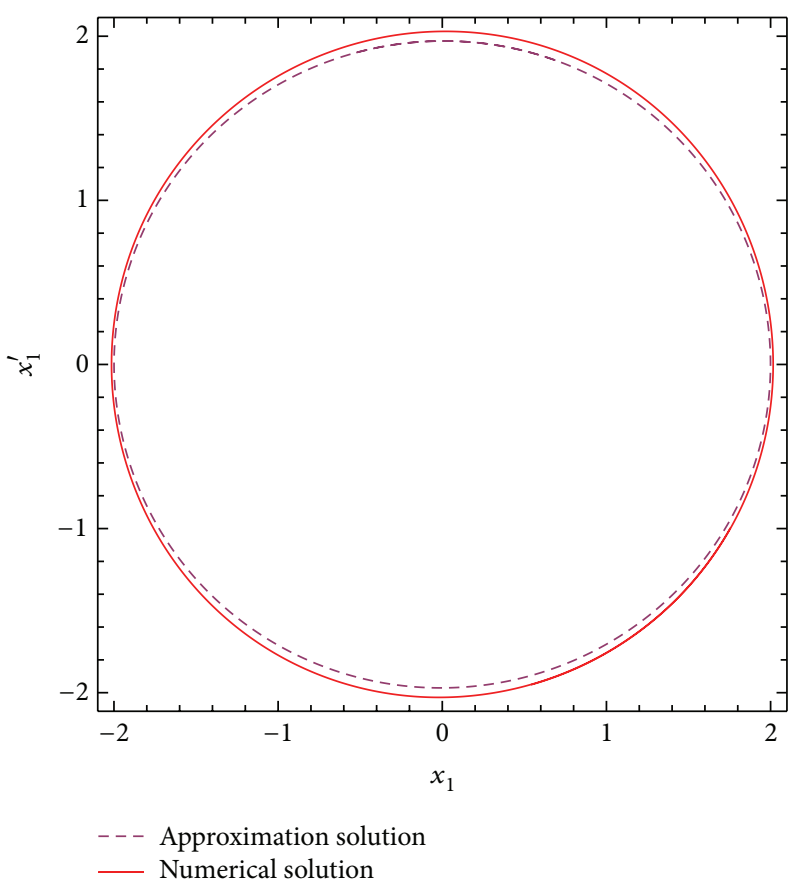

(a)

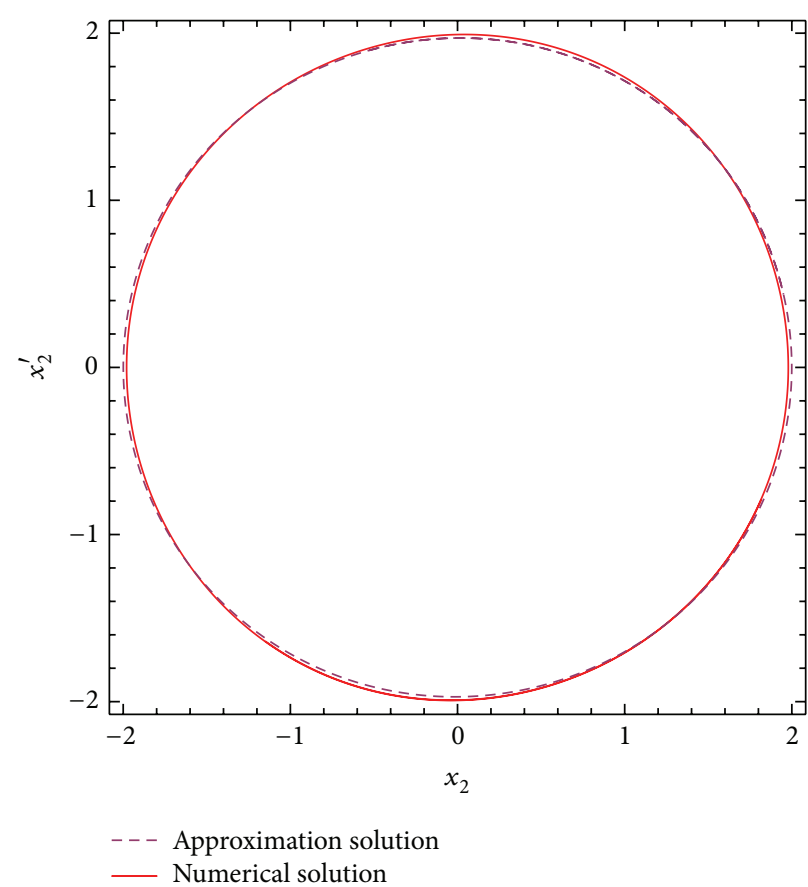

(b)

FIGURE 1: Comparison of the phase portrait curves of the forth-order approximation with the numerical integration method.

$$
\begin{aligned}
& \left(\begin{array}{l}
S_{1, m}\left(\tau, \vec{\omega}_{m}\right) \\
S_{2, m}\left(\tau, \vec{\omega}_{m}\right)
\end{array}\right) \\
& =\left(\begin{array}{c}
\left(\sum_{i=0}^{m} \omega_{i} \omega_{m-i}-\chi_{m} \sum_{i=0}^{m-1} \omega_{i} \omega_{m-1-i}\right)(\cos \tau+\sin \tau) \\
\left(\sum_{i=0}^{m} \omega_{i} \omega_{m-i}-\chi_{m} \sum_{i=0}^{m-1} \omega_{i} \omega_{m-1-i}\right) \sin \tau
\end{array}\right) \\
& +\left(Q_{m}\left(\vec{\delta}_{m}\right)-\chi_{m}\left(\vec{\delta}_{m-1}\right)\right) \\
& Q_{m}\left(\vec{\delta}_{m}\right)=\left(\begin{array}{c}
a_{m} \cos \tau+b_{m} \sin \tau \\
c_{m} \cos \tau
\end{array}\right)
\end{aligned}
$$

Because of the principle of solution expression and the linear operator $L$, the right side of (33) should not contain the terms of $\sin \tau$ and $\cos \tau$ or the secular terms $\tau \sin \tau$ and $\tau \cos \tau$. The coefficients are set to be zero to yield

$$
\begin{array}{r}
\frac{1}{\pi} \int_{0}^{2 \pi}\left[\hbar_{1} R_{1, m}\left(\vec{u}_{1, m-1}, \vec{\omega}_{m-1}\right)\right. \\
\left.+\hbar_{2} S_{1, m}\left(\tau, \vec{\omega}_{m}\right)\right] \cos \tau d \tau=0, \\
\frac{1}{\pi} \int_{0}^{2 \pi}\left[\hbar_{1} R_{1, m}\left(\vec{u}_{1, m-1}, \vec{\omega}_{m-1}\right)\right. \\
\left.+\hbar_{2} S_{1, m}\left(\tau, \vec{\omega}_{m}\right)\right] \sin \tau d \tau=0,
\end{array}
$$

$$
\begin{array}{r}
\frac{1}{\pi} \int_{0}^{2 \pi}\left[\hbar_{1} R_{2, m}\left(\vec{u}_{2, m-1}, \vec{\omega}_{m-1}\right)\right. \\
\left.+\hbar_{2} S_{2, m}\left(\tau, \vec{\omega}_{m}\right)\right] \cos \tau d \tau=0, \\
\frac{1}{\pi} \int_{0}^{2 \pi}\left[\hbar_{1} R_{2, m}\left(\vec{u}_{2, m-1}, \vec{\omega}_{m-1}\right)\right. \\
\left.+\hbar_{2} S_{2, m}\left(\tau, \vec{\omega}_{m}\right)\right] \sin \tau d \tau=0 .
\end{array}
$$

The solutions of $\omega_{k}, a_{k}, b_{k}$, and $c_{k}(k=0,1,2, \ldots)$ from (33) and (36a), (36b), (36c), and (36d) can be computed successively. To achieve more accurate results, we modify the solution of $\omega$ as follows:

$$
\bar{\omega}=\omega+v
$$

where $v$ is a small parameter.

\section{Numerical Simulation and Discussion}

In this section, numerical experiment is conducted to verify the accuracy of the present approach.

Taking $\varepsilon=1 / 100, \eta_{1}=1, \eta_{2}=2, \alpha_{1}=1 / 2, \alpha_{2}=1 / 3$, $\delta_{1}=2 / 10, \delta_{2}=3 / 10$, and the initial approximations of $a, b$, $c$, and $\omega$ are $a_{0}=2, b_{0}=0, c_{0}=2$, and $\omega_{0}=1087 / 1000$, respectively.

For simplicity and accuracy, we set $\hbar_{1}=-0.1, \hbar_{2}=300$, and $v=-0.1$; then the comparison of the phase curves of the fifth-order approximation with the numerical integration solution is shown in Figure 1. 


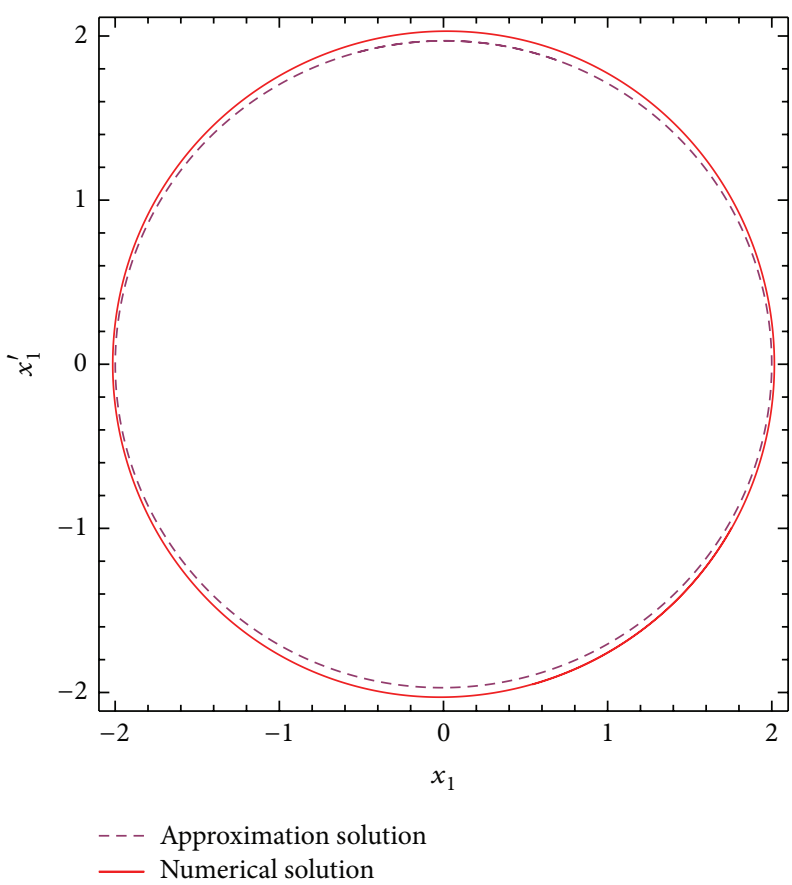

(a)

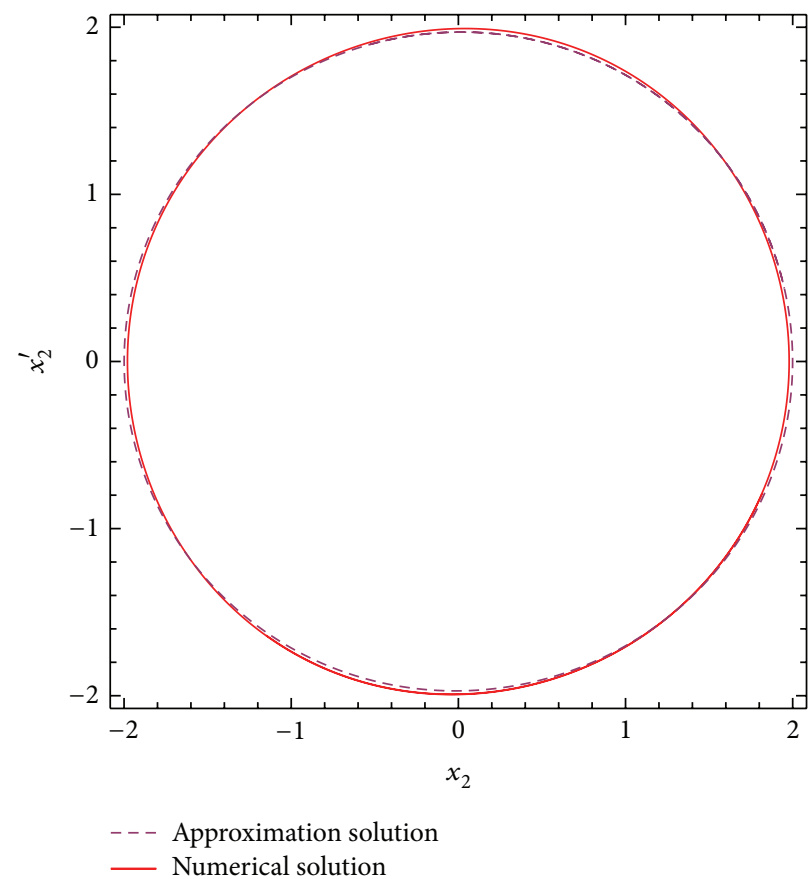

(b)

FIGURE 2: Comparison between the phase curves of the fifth-order approximation and the numerical integration out-of-phase solution.

The initial conditions of the numerical integration method are $x_{1}(0)=1.999538, x_{1}^{\prime}(0)=0.000037, x_{2}(0)=$ 1.999458 , and $x_{2}^{\prime}(0)=0$.

Moreover, the fifth-order analytical solutions $\left(x_{1}(t)\right.$, $x_{2}(t)$, and $\left.\omega\right)$ are given as

$$
\begin{aligned}
\begin{aligned}
& x_{1}(t)= 1.99884944 \cos \bar{\omega} t+0.00321365 \sin \bar{\omega} t \\
&+0.00068916 \cos 3 \bar{\omega} t-0.00106812 \sin 3 \bar{\omega} t, \\
& x_{2}(t)= 1.99883406 \cos \bar{\omega} t+0.00641377 \sin \bar{\omega} t \\
&+0.00062615 \cos 3 \bar{\omega} t-0.00213651 \sin 3 \bar{\omega} t, \\
& \omega=1.08696631,
\end{aligned}
\end{aligned}
$$

in which

$$
\bar{\omega}=\omega+v=0.98696631 .
$$

The above results demonstrate that the system has an inphase solution. While giving the initial approximations of $a_{0}=2, b_{0}=0, c_{0}=-2$, and $\omega_{0}=1087 / 1000$, we can get an out-of-phase solution to the system.

In this case, the comparison between the phase curves of the fifth-order approximation and the numerical integration solution is portrayed in Figure 2.

The initial conditions of the numerical integration method are $x_{1}(0)=1.999392, x_{1}^{\prime}(0)=0.000110, x_{2}(0)=$
-1.999458 , and $x_{2}^{\prime}(0)=0$, and the fifth-order analytical solutions are written as

$$
\begin{aligned}
\begin{aligned}
& x_{1}(t)= 1.99870293 \cos \bar{\omega} t+0.00309709 \sin \bar{\omega} t \\
&+0.00068900 \cos 3 \bar{\omega} t-0.00106809 \sin 3 \bar{\omega} t, \\
& x_{2}(t)=-1.99883388 \cos \bar{\omega} t-0.00641395 \sin \bar{\omega} t \\
&-0.00062613 \cos 3 \bar{\omega} t-0.00213658 \sin 3 \bar{\omega} t, \\
& \omega=1.08703369,
\end{aligned}
\end{aligned}
$$

in which

$$
\bar{\omega}=\omega+v=0.98703369 .
$$

\section{Conclusions}

In the present paper, the EHAM approach is applied to get asymptotic analytical series solutions of 2-DOF van der PolDuffing oscillators with a nonlinear coupling. The basic idea described in this paper is expected to be more employed in solving other dynamical systems in engineering and physical sciences. Comparisons with the numerical results are presented to demonstrate the validity of this method. In summary, compared with some other methods, the EHAM has the following advantages.

(1) The EHAM provides an ingenious avenue for controlling the convergences of approximation series. Numerical comparisons demonstrate that the EHAM is an effective and robust analytical method of 2-DOF van der Pol-Duffing oscillators. 
(2) Because of its flexibility, the present techniques can also be further generalized to analyze more complicated nonlinear MDOF dynamical systems that can only be analyzed by numerical methods.

\section{Conflict of Interests}

The authors declare that there is no conflict of interests regarding the publication of this paper.

\section{Authors' Contribution}

All the authors contributed equally and significantly to the writing of this paper. All the authors read and approved the final paper.

\section{Acknowledgments}

The author Y. H. Qian gratefully acknowledges the support of the National Natural Science Foundations of China (NNSFC) through Grants nos. 11202189 and 11304286 and the Natural Science Foundation of Zhejiang Province of China through Grant no. LY12A02002. The author S. M. Chen gratefully appreciates the financial support from the NNSFC through Grants no. 11371326. The author L. Shen gratefully acknowledges the support from open experiment project of Zhejiang Normal University. The authors are also grateful to the anonymous reviewers for their constructive comments and suggestions.

\section{References}

[1] R. E. Mickens, Mathematical Methods for the Natural and Engineering Sciences, World Scientific, Singapore, 2004.

[2] Y. K. Cheung, S. H. Chen, and S. L. Lau, "A modified LindstedtPoincaré method for certain strongly nonlinear oscillators," International Journal of Non-Linear Mechanics, vol. 26, no. 3-4, pp. 367-378, 1991.

[3] M. Senator and C. N. Bapat, "A perturbation technique that works even when the nonlinearity is not small," Journal of Sound and Vibration, vol. 164, no. 1, pp. 1-27, 1993.

[4] P. Amore and A. Aranda, "Improved Lindstedt-Poincaré method for the solution of nonlinear problems," Journal of Sound and Vibration, vol. 283, no. 3-5, pp. 1115-1136, 2005.

[5] R. R. Pušenjak, "Extended Lindstedt-Poincare method for non-stationary resonances of dynamical systems with cubic nonlinearities," Journal of Sound and Vibration, vol. 314, no. 12, pp. 194-216, 2008.

[6] J. L. Summers and M. D. Savage, "Two timescale harmonic balance. I. Application to autonomous one-dimensional nonlinear oscillators," Proceedings of the Royal Society of London A. Mathematical, Physical and Engineering Sciences, vol. 340, no. 1659, pp. 473-501, 1992.

[7] B. Wu and P. Li, "A method for obtaining approximate analytic periods for a class of nonlinear oscillators," Meccanica, vol. 36, no. 2, pp. 167-176, 2001.

[8] S. S. Ganji, D. D. Ganji, Z. Z. Ganji, and S. Karimpour, "Periodic solution for strongly nonlinear vibration systems by He's energy balance method," Acta Applicandae Mathematicae, vol. 106, no. 1, pp. 79-92, 2009.
[9] I. Mehdipour, D. D. Ganji, and M. Mozaffari, "Application of the energy balance method to nonlinear vibrating equations," Current Applied Physics, vol. 10, no. 1, pp. 104-112, 2010.

[10] L. Zhao, "He's frequency-amplitude formulation for nonlinear oscillators with an irrational force," Computers \& Mathematics with Applications, vol. 58, no. 11-12, pp. 2477-2479, 2009.

[11] J. Fan, "He's frequency-amplitude formulation for the Duffing harmonic oscillator," Computers \& Mathematics with Applications, vol. 58, no. 11-12, pp. 2473-2476, 2009.

[12] P. J. Hilton, An Introduction to Homotopy Theory, Cambridge University Press, Cambridge, UK, 1953.

[13] C. Nash and S. Sen, Topology and Geometry for Physicists, Academic Press, London, UK, 1983.

[14] S. J. Liao, The proposed homotopy analysis techniques for the solution of nonlinear problems [Ph.D. thesis], Shanghai Jiao Tong University, Shanghai, China, 1992.

[15] S. J. Liao, "A kind of approximate solution technique which does not depend upon small parameters. II. An application in fluid mechanics," International Journal of Non-Linear Mechanics, vol. 32, no. 5, pp. 815-822, 1997.

[16] S. Liao, Beyond Perturbation: Introduction to the Homotopy Analysis Method, Chapman \& Hall, Boca Raton, Fla, USA, 2004.

[17] S. Rajasekar and K. Murali, "Resonance behaviour and jump phenomenon in a two coupled Duffing-van der Pol oscillators," Chaos, Solitons and Fractals, vol. 19, no. 4, pp. 925-934, 2004.

[18] B. T. Nohara and A. Arimoto, "Non-existence theorem except the out-of-phase and in-phase solutions in the coupled van der Pol equation system," Ukrainian Mathematical Journal, vol. 61, no. 8, pp. 1311-1337, 2009.

[19] Y. J. Li, B. T. Nohara, and S. J. Liao, "Series solutions of coupled van der Pol equation by means of homotopy analysis method," Journal of Mathematical Physics, vol. 51, no. 6, pp. 1-12, 2010.

[20] Y. H. Qian, C. M. Duan, S. M. Chen, and S. P. Chen, "Asymptotic analytical solutions of the two-degree-of-freedom strongly nonlinear van der Pol oscillators with cubic couple terms using extended homotopy analysis method," Acta Mechanica, vol. 223, no. 2, pp. 237-255, 2012. 


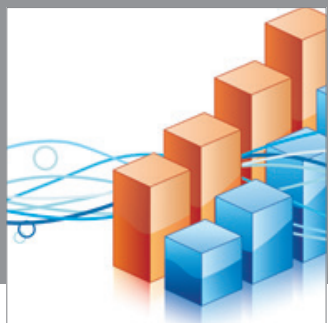

Advances in

Operations Research

mansans

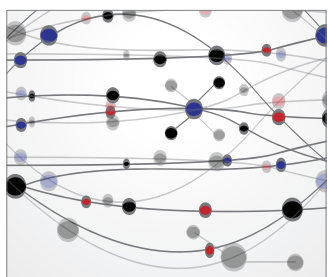

The Scientific World Journal
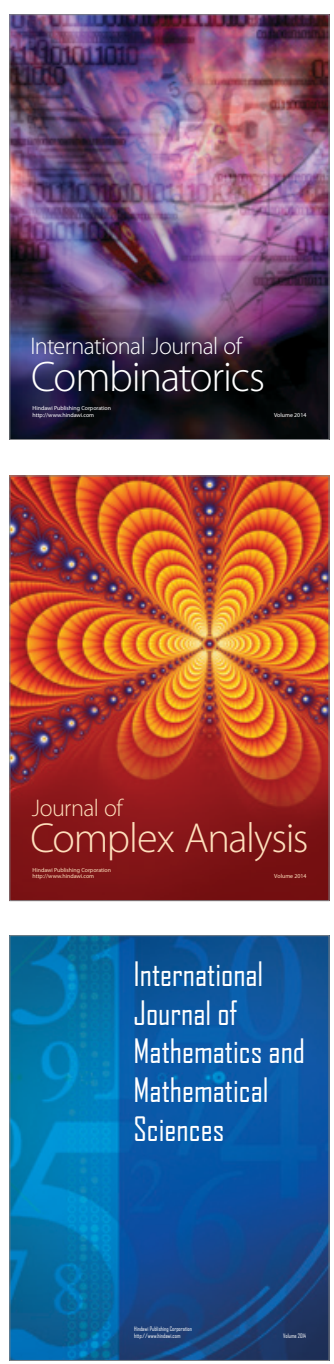
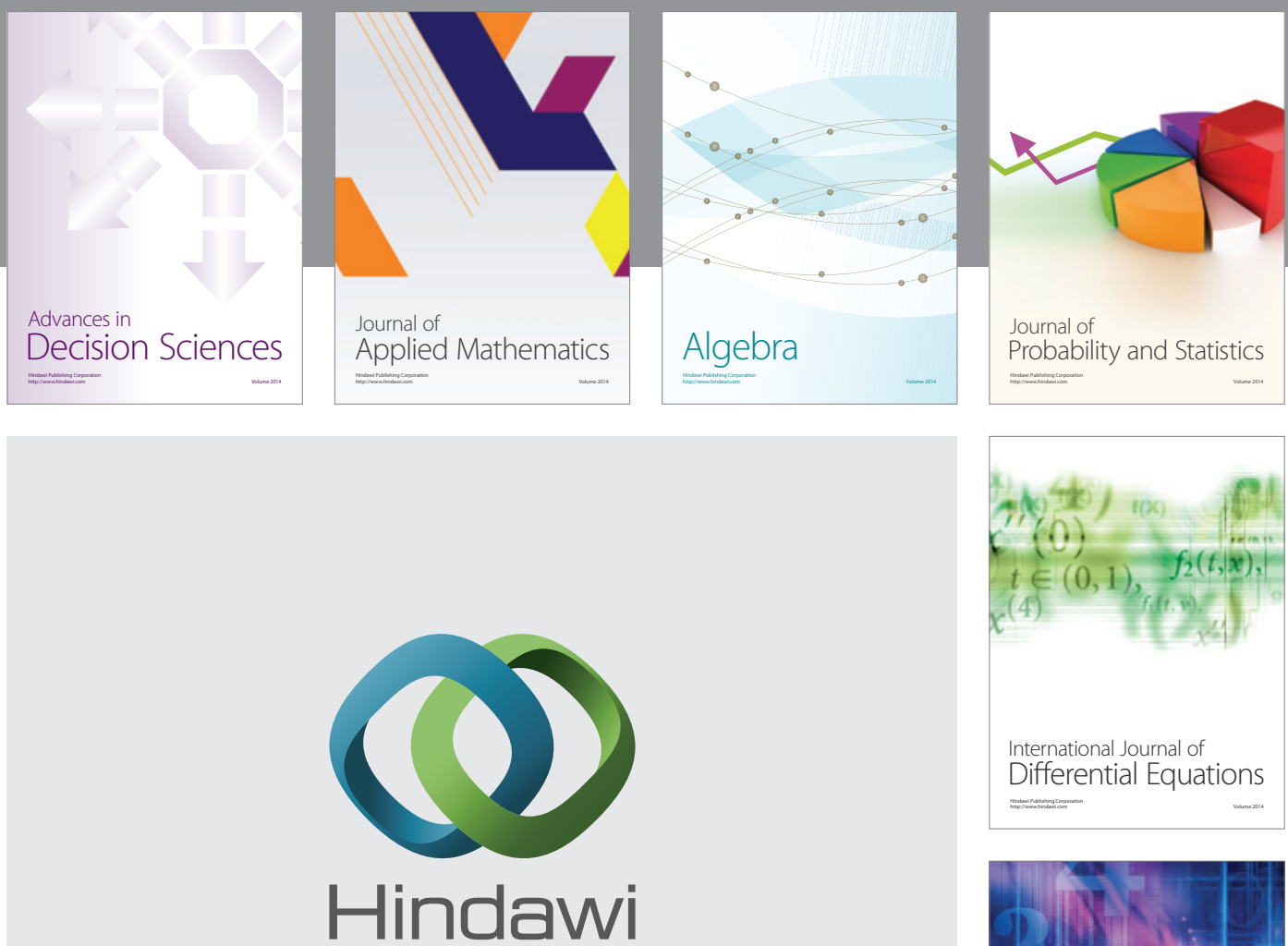

Submit your manuscripts at http://www.hindawi.com
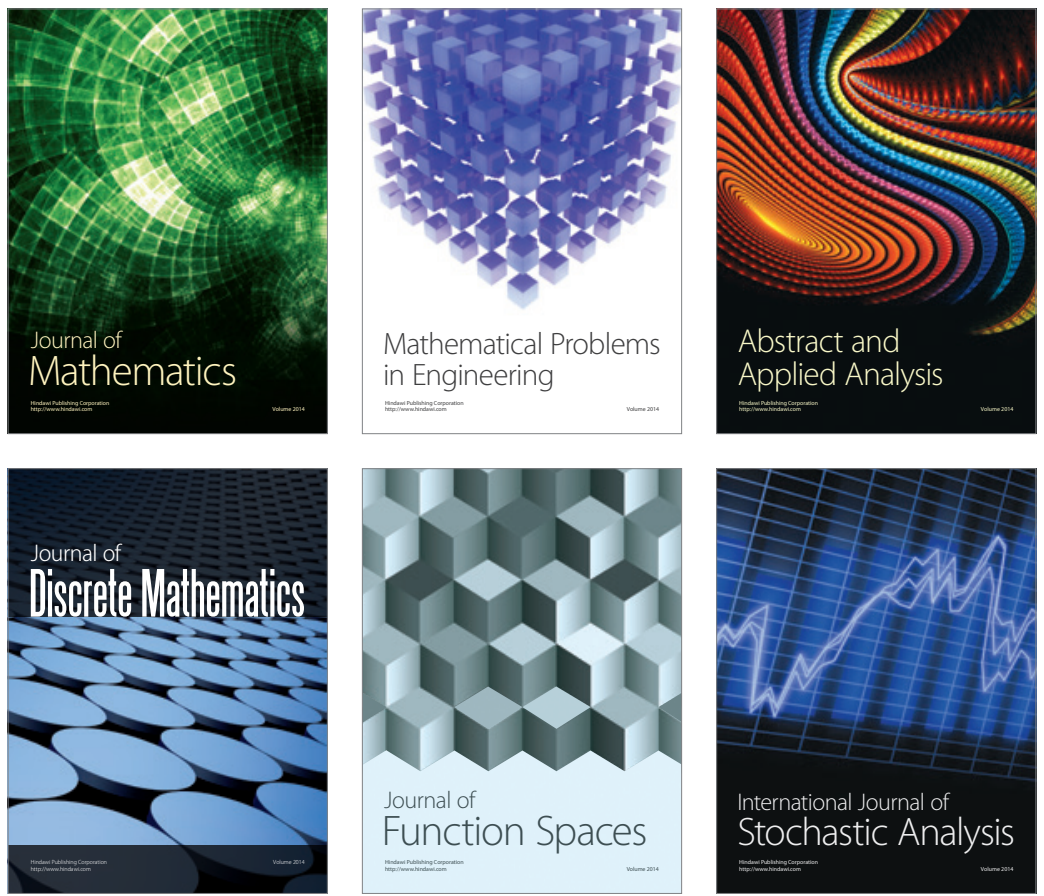

Journal of

Function Spaces

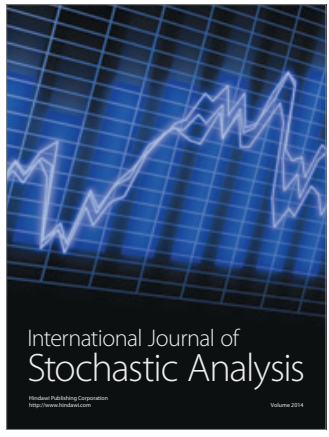

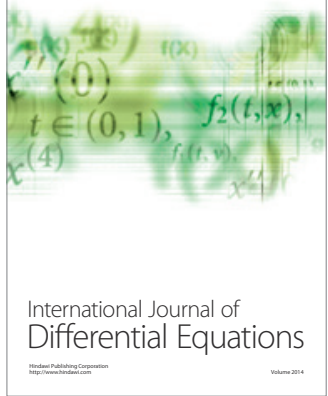
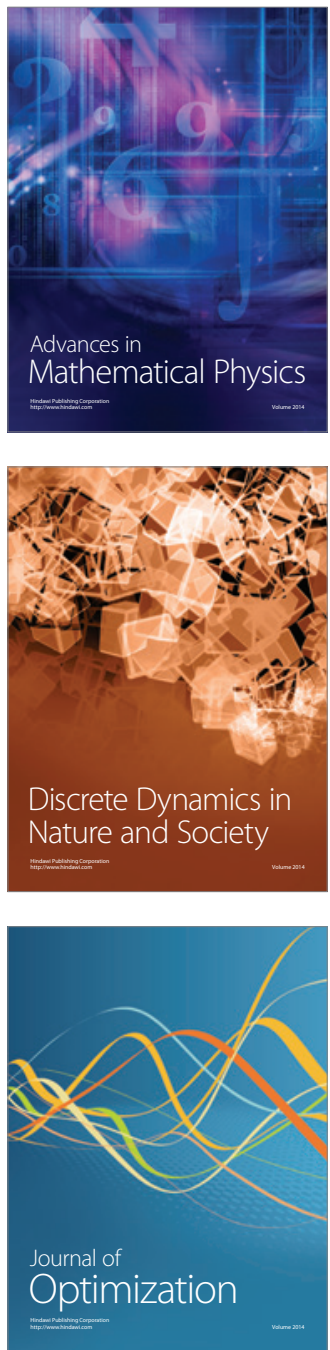